\title{
Der papierene Brief, der elektronische Brief (E-Mail) und deren Stellung und Sprache(n) in der internen Kommunikation multinationaler Unternehmen in Tschechien
}

\author{
Kateřina Šichová
}

This essay deals with the internal written communication in multinational (German-Czech) companies in the Czech Republic. The focus concentrates on the medium letter, which includes both the classical written letter and emails. The appearance of electronic post service and its rapid establishment shifted the role of the classical letter. In the meantime emails play an important role in the everyday life as well as in business communication, primarily due to its time savings. Compared to letters sent by post or fax machine, emails show some linguistic features. In terms of the used (foreign) languages and the translation of contents, emails subject to other strategies and take up a particular position in the intra-enterprise communication. For vivid reasons and more significance, the theses are proven by empirically determined economic data.

multinational corporations - internal communications - letter - e-mail - translation - foreign language - German - Czech - language management

Der Aufsatz beschäftigt sich mit der internen schriftlichen Kommunikation in multinationalen (deutsch-tschechischen) Unternehmen in Tschechien. Der Fokus liegt auf dem Medium Brief (klassischer Brief, E-Mail). Mit dem Aufkommen und der raschen Etablierung der elektronischen Post änderte sich die Rolle des klassischen (papierenen) Briefs. Die E-Mails spielen nicht nur in der privaten, sondern auch in der geschäftlichen Kommunikation eine wesentliche Rolle und weisen (z. B. aufgrund des beinahe zeitgleichen Sendens und Empfangens) einige sprachliche Unterschiede im Vergleich zum klassischen Brief auf (mediale vs. konzeptionelle Schriftlichkeit bzw. Mündlichkeit). Auch im Hinblick auf die verwendeten (Fremd)sprachen oder die Übersetzung der Inhalte unterliegen die E-Mails anderen Strategien und nehmen eine andere Stellung in der innerbetrieblichen Kommunikation ein. Die Aussagen im Aufsatz stützen sich auf empirische Daten aus der Wirtschaft.

multinationales Unternehmen - interne Kommunikation - mediale Schriftlichkeit konzeptionelle Schriftlichkeit - mediale Mündlichkeit - konzeptionelle Mündlichkeit - Brief E-Mail - Übersetzen - Fremdsprache - Deutsch - Tschechisch - Sprachmanagement

\section{Einleitung}

Seit langem ist es unumstritten, dass für den wirtschaftlichen Erfolg eines Unternehmens die erfolgreiche Kommunikation ausschlaggebend ist, sei es die Kommunikation nach außen, mit unterschiedlichen externen Zielgruppen (Geschäftspartner, Kunden, Lieferanten, Politik, Medien usw.), als auch die sog. interne Kommunikation innerhalb des Betriebes. 
Im Rahmen der Unternehmenskommunikation hat die schriftliche Kommunikation (v. a. Briefe) stets eine enorm wichtige Stellung genossen. ${ }^{1}$

Seit dem Aufkommen der elektronischen Medien hat jedoch die schriftliche verbale Kommunikation (formelle wie auch informelle) schlagartige Änderungen erfahren; dabei scheint die E-Mail die entscheidendste Rolle zu spielen. Dies hat(te) auch für die mündliche Kommunikation etliche Konsequenzen. Durch vermehrte Nutzung medialer Kommunikationssysteme bekommt in der betrieblichen Kommunikation der klassische Brief ${ }^{2}$ allmählich eine andere Rolle, als er früher hatte. Auch die Unterschiede in Formalien oder im Sprachgebrauch beider Kommunikate (klassischer Brief, elektronischer Brief) werden immer größer. Auswirkungen hat die Dominanz der E-Mail in einigen betrieblichen Kommunikationssphären ebenfalls auf die Verwendung von (Fremd)sprachen in der schriftlichen und mündlichen Kommunikation.

Im Folgenden sollen einige Überlegungen zu den E-Mails als Teil der innerbetrieblichen schriftlichen Kommunikation im Hinblick auf das Phänomen des klassischen Briefs in den Unternehmen geliefert werden. Illustriert werden soll die Problematik an der Korrespondenz ${ }^{3}$ einer speziellen Gruppe von Unternehmen, den sog. multinationalen Unternehmen, die spätestens seit der Zeit der Globalisierung auf der ganzen Welt in zunehmendem Maße entstehen. Es handelt sich um (in verschiedener Form und unterschiedlichem Ausmaß) ,gemischte" Unternehmen, in welchen die Gestaltung der Kommunikation mit zusätzlichen Faktoren konfrontiert wird, wie beispielsweise dem Faktor (Fremd)Sprache. ${ }^{4}$ Ins Visier genommen werden hier die deutsch-tschechischen Unternehmen auf dem Gebiet der Tschechischen Republik und die Daten aus diesen Firmen sollen den Thesen und Darstellungen eine größere Aussagekraft verleihen. ${ }^{5}$

Aus der Sicht der Interkulturalität zeichnet sich hierbei eine Grundsatzfrage ab: Werden im Rahmen der schriftlichen internen Kommunikation in den deutsch-tschechischen Unternehmen in Tschechien neben Tschechisch auch

1 Vgl. einige Managementstrategien und Sprachenplanung der Firmen; für multinationale Unternehmen z. B. in NEKVAPIL/SHERMAN (2008) oder VOLLSTEDT (2002).

2 Darunter verstehe ich zum Zwecke der für diesen Aufsatz ausschlaggebenden Unterscheidung vom elektronisch verfassten und verschickten Brief (E-Mail) einen auf Papier festgehaltenen und per Post bzw. per Fax verschickten Brief. Genau genommen würde der gefaxte Brief auch nicht unter den klassischen Brief fallen, da er der Eigenart des Transportes des Briefs nicht gerecht ist (er steckt nicht in einem geschlossenen Briefumschlag und ist nicht frankiert oder anderweitig markiert). Auf diese Spezifika wird hier jedoch nicht weiter eingegangen.

3 Zum Begriff vgl. HLADKÁ $(2006,5)$. Primär drückt das Wort ein briefliches Agieren zwischen einem Emittenten und einem Adressaten, sekundär (und in diesem Sinne wird es im Folgenden verwendet) dann das Korpus von Korrespondenztexten.

4 Zur Mehrsprachigkeit in der Wirtschaft vgl. BUNGARTEN (1997).

5 Allerdings muss betont werden, dass jedes Unternehmen auch bei der Gestaltung, Regulierung und Durchführung der Kommunikation über etliche Spezifika verfügt, so dass bei jeder Pauschalisierung äußerste Vorsicht geboten ist. 
Fremdsprachen (Deutsch und/oder Englisch) verwendet? Und wenn ab einer bestimmten Hierarchieebene Tschechisch überhaupt verwendet wird, in welchen Situationen? Kommen alle Mitarbeiter mit ihren Sprachkenntnissen aus oder müssen Sprachvermittler eingesetzt werden?

Die Überlegungen zu Briefen bzw. E-Mails gehen dann in drei Richtungen: 1. Wird der elektronische Brief in den Unternehmen genauso (d. h. in denselben Kommunikationssituationen, gleich häufig usw.) eingesetzt wie der klassische Brief? 2. Unterscheidet sich die innerbetriebliche E-Mail-Kommunikation in sprachlicher Hinsicht von der Kommunikation mittels klassischer Briefe (d. h. werden dieselben Inhalte/Themen vermittelt, werden dieselben sprachlichen Mittel eingesetzt, ist die Funktion gleich)? 3. Werden mehrere (und wenn ja, welche bzw. gleichzeitig mehrere) Sprachen in der E-Mail-Kommunikation im Unternehmen verwendet und wenn ja, wann?

\section{Schlüsselbegriffe, Materialgrundlage}

Zunächst sollen die Wörter Brief, E-Mail, interne Kommunikation angesprochen werden, anschließend wird die Materialgrundlage (befragte Unternehmen, Datenerhebung) kurz skizziert.

\subsection{Brief}

Für die Zwecke dieses Aufsatzes wird Briefals eine bestimmte Kommunikationsform betrachtet. ${ }^{1}$ Aus der Sicht des Handlungsbereiches kann mein Untersuchungsgegenstand unter die Geschäftsbriefe ${ }^{2}$ eingeordnet werden. Wie jedoch bei den weiteren Ausführungen ersichtlich wird, kommt es gerade bei dem per E-Mail verschickten elektronischen Brief manchmal zu einer Vermischung des Offiziellen und des Privaten.

1 Es kann hier, unter Verweis auf einschlägige Literatur (z. B. NICKISCH: 1991; ERMERT: 1979), nicht auf alle brieftheoretischen Bemühungen eingegangen werden, zumal einige wesentlichen definitorischen und begrifflichen Fragen in der Forschung bis dato nicht geklärt sind (vgl. HLADKÁ: 2006, 16). Zu den Grundfunktionen des Briefs als Text vgl. NICKISCH (1991, 13). Aus dem natürlichen Ablauf der Kommunikation ergibt sich der (in aller Regel vorhandene) formale Ablauf eines Briefes mit den Grundbestandteilen Briefeingang, Briefinhalt, Briefschluss (vgl. ERMERT: 1979, 104). Das Erstere und Letztere neigen zur Formalisierung und können somit als Eingangsund Schlussformeln (Anrede, Grüße, Unterschrift) unter gewissen Umständen ausgelassen bzw. fest vorformuliert werden. Siehe auch weiter unten.

2 Von diesen sondert sich die andere Hauptgruppe ab, die Privatbriefe. Die Gestaltung von Geschäftsbriefen wird, was die äußere Form anbelangt, in Deutschland durch die DIN-Norm Nr. 5008 und Nr. 676 (des Deutschen Instituts für Normung) geregelt, vgl. auch GRÜN (2005). Die Grundlagen der allgemeinen formalen Gestaltung sind in Tschechien in den Normen ČSN 886101 und ČSN 016910 verankert, für die Gestaltung der Anschriftenfelder ist auch das Gesetz Nr. 29/2000 der Sammlung von Bedeutung. 


\subsection{E-Mail}

Auch die E-Mail ${ }^{1}$ ist eine Kommunikationsform, sie übernimmt und variiert die Textgestalt des klassischen Briefes (GREULE: 2008). ${ }^{2}$ Das technische Format der E-Mails wird durch eine Art Norm festgelegt (Protokoll RFC 2822). ${ }^{3}$ Formal gesehen muss eine E-Mail aus zwei Teilen bestehen, dem sog. Header (u. a. Angaben zu Absender, (Kopie-)Empfänger, Datum, Betreff-Zeile) und dem sog. Body (Brieftext und Anhänge).

Das wichtigste Markenzeichen dieser schriftlichen Nachricht ist, dass sie im Zusammenspiel mit elektronischen Geräten gefertigt wird ${ }^{4}$ und auf elektronischem Wege in Computernetzwerken übertragen wird. Daraus resultieren auch einige formale Unterschiede zum auf postalischem Wege verschickten Brief. Zum Beispiel ist der (mit der zumindest teilweisen räumlichen Trennung verbundene) zeitliche Phasenverzug zwischen der Absendung und dem Empfang zwar nach wie vor gegeben, aufgrund der Tatsache aber, dass heutzutage die Laufzeit der E-Mail weltweit bei ungestörten Bedingungen selten mehr als eine Minute beträgt, ändern sich die zeitlichen Dimensionen. Die E-Mail ist also zwar nach wie vor ein asynchrones Kommunikationsmedium, nähert sich jedoch dem zeitgleichen Senden und Empfangen, der „Face-to-face-Kommunikation“ in erheblichem Maße an. All das erzeugt bei den Kommunikationsteilnehmern die „Perzeption von Nähe“ (STEIN: 2002, 314), die sowohl positiv als auch negativ empfunden werden kann und sich u. a. in sprachlichen Formen manifestiert, vgl. weiter unten oder HLADKÁ (2006, 144 ff.). Aus diesem Grund wird manchmal die E-Mail als eine Art Zwischenweg zwischen der schriftlichen und der mündlichen Kommunikation wahrgenommen und genutzt, was sich wiederum in einer Neubesetzung der Rolle des klassischen Briefes zeigt, vgl. weiter unten.

1 Die Bezeichnung E-Mail kommt aus dem Englischen und ließe sich auf Deutsch als, die elektronische Post' oder ,der elektronische Brief' ausdrücken (in diesem Sinne wird das Wort in dieser Arbeit verwendet).

2 Die Diskussion diesbezüglich, ob eine E-Mail eine Art Brief ist und warum, lasse ich hier weitgehend außer Acht. Dafür, dass die E-Mail ein Brief ist, spricht nicht nur der briefähnliche Aufbau, Kommunikationsablauf oder dieselben Grundfunktionen, sondern auch die Tatsache, dass die E-Mail von der Gesetzgebung im Hinblick auf seine Verbindlichkeit im Rechtsverkehr, das Briefgeheimnis oder das Urheberrecht wie ein Brief angesehen wird: Sie setzt „die E-Mail einem verschlossenen Brief gleich“ (vgl. Urteil vom 6. 9. 2006, LG Köln zum Az.: 28 O 178/06); weiteres hierzu siehe z. B. unter http://www.online-werberecht.de/emailbeweis.html (letzter Zugriff am 26. 07. 2010).

3 Weiteres unter http://www.teialehrbuch.de/Kostenlose-Kurse/Internet-Technik/16293-RFC-2822Internet-Message-Format.html (letzter Zugriff am 26. 07. 2010).

4 Man spricht vom Konzept der elektronischen Schriftlichkeit, d.h. computermediierten Form der schriftlichen Textproduktion, vgl. GÜNTHER KLEINBERGER/THIMM $(2000,5)$. 


\subsection{Interne Kommunikation}

Unter der internen Kommunikation ${ }^{1}$ wird hier die Kommunikation zwischen den Angehörigen eines Unternehmens im Rahmen dieses Unternehmens verstanden. Sie hat den Sinn und Zweck des Austausches, der Informationsverbreitung und nicht zuletzt der Optimierung organisatorischer Abläufe, der Effizienz, und verläuft auf formellem und informellem Weg.

Für die formelle Kommunikation sind im Unternehmen bestimmte Rahmenbedingungen vorgegeben und sie ist nur bedingt frei gestaltbar, vielmehr determiniert durch organisatorische Vorgaben, Regeln für die Form, den Inhalt und den Ablauf der Kommunikation. Hierzu kann auch die Regulierung der Sprache in vielerlei Hinsicht gehören, die sich in manchen multinationalen Unternehmen z. B. in der Einführung der sog. Firmensprache manifestiert. Somit ist eines der wichtigsten Merkmale dieser Kommunikationsart eine gewisse Personen-Unabhängigkeit, die jedoch, wie später gezeigt wird, gerade in der E-Mail-Kommunikation relativiert wird. Zu den weiteren Merkmalen gehört die Schriftlichkeit oder Verschriftlichung (z. B. Protokolle aus den Mitarbeitergesprächen etc.).

Sowohl die informelle als auch die formelle (interne) Kommunikation kann schriftlicher oder mündlicher Natur sein.

Im Weiteren konzentriere ich mich nur auf den schriftlichen Teil dieser Kommunikationsart. Für die Abwicklung der internen schriftlichen Kommunikation stehen mehrere Medien zur Verfügung, zu den klassischen gehören z. B. Rundschreiben, Mitarbeiterzeitschrift, Brief, schwarzes Brett, Mitarbeitergespräch, Betriebsversammlung; zu den elektronischen dann beispielsweise E-Mail, Intranet, Online-Newsletter. Kombiniert werden dabei die sog. Push-Instrumente, also Medien, die Informationen direkt an einen bestimmten Empfänger oder eine Empfängergruppe transportieren, wozu der Brief oder die E-Mail gehören, und die sog. Pull-Instrumente, die Informationen auf eine Plattform stellen, von wo diese aktiv abgerufen werden müssen.

\subsection{Materialgrundlage: Multinationale Unternehmen in Tschechien}

In den weiteren Ausführungen möchte ich versuchen, die Brief-/Mail-Problematik im Kontext einer konkreten Unternehmensgruppe zu betrachten, nämlich der in der Tschechischen Republik angesiedelten gemischten deutsch-tschechischen ${ }^{2}$

1 Zum Begriff Kommunikation vgl. HERINGER (2007, 10 ff.) bzw. GLAHN (2005, 15 ff.). Auf die nonverbale Kommunikation kann hier nicht näher eingegangen werden, obgleich sie eine wichtige Rolle spielt.

2 In der unten vorgestellten Untersuchung wurden auch österreichisch-tschechische und schweizertschechische Unternehmen in Tschechien berücksichtigt. Somit steht hier der Ausdruck „deutschtschechische Unternehmen" auch als eine Art Abkürzung für alle diese Firmen und akzentuiert die Sprachdimension („deutschsprachig“). Zur Anzahl solcher Firmen in der Tschechischen 
Unternehmen. ${ }^{1}$ Diese Unternehmen sind bereits per definitionem bi- (bzw. multi-) national, also bi- (bzw. multi-) kulturell, was für ihre Mitarbeiter zahlreiche Spezifika im Hinblick auf die wirtschaftliche, soziokulturelle und sprachliche Situation mit sich bringt. Betont werden muss aber noch, dass es sich hier - aus der Sicht des Kapitalengagements - sowohl um Firmen, d. h. Tochtergesellschaften oder Betriebstätten, mit 100\%-iger deutscher Beteiligung, aber auch um verschiedene andere Formen von "Mischgesellschaften “2 mit unterschiedlich hoher Kapitalbeteiligung handelt; selbstverständlich differiert auch die Größ $\mathrm{e}^{3}$ der Unternehmen.

Mein Material stammt zum Teil aus den im Rahmen des FOROST-Projektes ${ }^{4}$ gewonnenen Daten, sowohl aus den quantitativ analysierten Fragebogen als auch aus einigen teilstrukturierten Interviews, die qualitativ untersucht wurden. Zweitens wurden aus Anlass dieses Aufsatzes Analysen von papierenen Briefen bzw. Faxen und E-Mails aus einem deutsch-tschechischen Unternehmen durchgeführt und bereits fertige E-Mail-Analysen von einem anderen Unternehmen studiert. Weiterhin wurden zwecks Optimierung und Aktualisierung der Erkenntnisse einige informelle Gespräche mit den in diesen Unternehmen tätigen Mitarbeitern durchgeführt und auch E-Mails und andere schriftliche Unterlagen gesichtet.

\section{Sprachvermittlung in der internen schriftlichen Kommunikation in den Unternehmen}

Im Weiteren wird die schriftliche interne Kommunikation der deutschtschechischen Unternehmen fokussiert, zu welcher ich hier ausdrücklich auch die Kontakte der Tochtergesellschaft in Tschechien zum Mutterunternehmen in Deutschland zähle. ${ }^{5}$

Republik bzw. ihrem wirtschaftlichen Anteil an ausländischen Direktinvestitionen in Tschechien vgl. ŠICHOVÁ (2008, 219 f.), NEKULA/MARX/ŠICHOVÁ (2009, 62 ff.).

1 Zur Forschungsgeschichte der Sprachsituation in der Wirtschaft vgl. NEKULA/NEKVAPIL/ ŠICHOVÁ (2005a). Zu Sprachkontakt in multinationalen Betrieben vgl. AMMON (1997). Zur Sprachenplanung im tschechischen Gesamtkontext vgl. NEUSTUPNÝ/NEKVAPIL (2006).

2 Dementsprechend unterschiedlich werden dort auch das Ausmaß von „fremden“/,einheimischen“ Kulturstandards, Führungs-, Kommunikations- und Arbeitsstils und die Rolle der tschechischen Sprache bzw. der Fremdsprachen in der Unternehmenskommunikation sein, wie auch das Ausmaß der Regulierung der Kommunikation in der Firma.

3 Ich teile die Unternehmen nach der Mitarbeiterzahl auf: als klein wird ein Unternehmen mit weniger als 10 Beschäftigten betrachtet, ein mittelgroßes hat zwischen 10 und 499 Mitarbeiter, ein großes 500 und mehr. Diese Definition richtet sich nach den Angaben der statistischen Berichte über die Unternehmensgrößenstatistik des Bundesministeriums für Wirtschaft und Technologie, 1997/98 (vgl. MÖLLER/BRANDMEIER: 2002, 42).

4 Ausführlicher zum Projekt siehe NEKULA/NEKVAPIL/ŠICHOVÁ (2005a).

5 Es ist wichtig, die Einschränkung auf die Kommunikationsprozesse innerhalb des Unternehmens im Auge zu behalten, da, trotz vieler Gemeinsamkeiten, die Kommunikation und Auftritte der Firmen nach außen sicherlich anderen Regeln und Bedingungen unterliegen, z. B. den Wünschen der Kunden, diversen Anforderungen von Behörden, der Gesetzgebung des tschechischen Staats usw., vgl. NEKULA/MARX/ŠICHOVÁ (2009, 55 ff.). 
Verwendet werden hierbei (so gut wie ausschließlich) die Sprachen Tschechisch, Deutsch und Englisch, in verschiedenen Situationen in unterschiedlichem Maße; vgl. auch NEKULA/NEKVAPIL/ŠICHOVÁ (2005a).

Mit ihrer Internationalisierung und somit entstandener Mehrsprachigkeit müssen also multinationale Unternehmen bzw. deren Mitarbeiter für jede Kommunikationssituation einen (und zwar den richtigen) Sprachmodus wählen. Im Rahmen der Optimierung der Unternehmenskommunikation haben so manche Unternehmen auch auf eine Art Regulierung der Sprachverwendung zurückgegriffen, indem sie z. B. (insbesondere für den schriftlichen Verkehr) die so genannte Firmensprache ${ }^{1}$ festlegen oder die Wahl einer Sprache empfehlen.

Zitat $^{2} 1$ (Fragebogen):

Die Firmensprache ist Deutsch, nach Bedarf auch Englisch.

Zitat 2 (Interview Dt.):

KŠ: Ist in Ihrer unternehmensinternen Kommunikation eine Sprache als Firmensprache vorgegeben?

Befr.: Jein. [...] Nachdem wir ein Unternehmen sind, das international tätig ist, haben wir eine nicht festgelegte Unternehmenssprache, mit der wir uns auf der ganzen Welt verständigen können, und das ist Englisch. Aber sie ist nicht festgelegt.

Dass es - trotz Deklarierungen von Firmensprachen u. ä. - in gemischten Unternehmen aber häufig schwierig ist, für diverse Kommunikationssituationen die optimale Kommunikationssprache zu finden, ${ }^{3}$ und dass es manchmal in den Unternehmen zu Kommunikationsdominanz bzw. Subdominanz kommt (bzw. zur sprachlichen Asymmetrie, vgl. VANDERMEEREN: 1998), zeigen beispielsweise die Angaben hinsichtlich der Tschechischkenntnisse der deutschen Mitarbeiter,

1 Auffallend ist, dass - obwohl die Mitarbeiter diesen Begriff kennen und selber verwenden - es kaum möglich ist, eine zufriedenstellende „Definition“ der Firmensprache in den öffentlichen (Internet)Präsentationen der Unternehmen zu finden bzw. von den Unternehmen - häufig mit Verweis auf den internen Charakter solcher Angelegenheiten - zu bekommen. Somit sind die Forscher aber in mancher Hinsicht nur auf Interpretationen angewiesen, was freilich ein Risiko in sich birgt. Ausführlicher vgl. ŠICHOVÁ (2008).

2 Alles, was in diesem Aufsatz aus Fragebogen, Interviews oder gesichteten Briefen und E-Mails zitiert wird, steht in Kursivschrift. Tschechische Texte oder Interview-Transkriptionen (zu den Regeln vgl. NEKULA/NEKVAPIL/ŠICHOVÁ: 2005b) wurden bis auf Ausnahmen von mir ins Deutsche übersetzt und die Transkription vereinfacht. Die Verzerrung, die dadurch entsteht, nehme ich für die Zwecke dieses Aufsatzes in Kauf.

3 Es darf auch nicht vergessen werden, dass jede Sprache in unterschiedlichen Kontexten mit bestimmten „Rollen“ besetz ist. So sind die Fremdsprachen (hier primär das Deutsche) die Sprache(n) der Führungsebene, der Muttergesellschaft, der Geldgeber usw. Diese Sprachen sind oder waren Träger des „westlichen“ Know-hows usw. Dieser durchaus wichtige und interessante Faktor wird hier jedoch außer Acht gelassen. 
der Deutsch- und Englischkenntnisse der tschechischen Mitarbeiter ${ }^{1}$ bzw. des Sprachkursangebotes für die Mitarbeiter des Unternehmens (vgl. NEKULA/ NEKVAPIL/ŠICHOVÁ: 2005a) und vor allem Angaben über die stattfindende Sprachvermittlung.

Das Ausmaß an Sprachvermittlung allgemein ist in den multinationalen Unternehmen groß, vgl. NEKULA/ŠICHOVÁ (2004). Wenn man zuerst nach dem Ausmaß des Übersetzens der Sprachen Deutsch-Tschechisch für die internen Bedürfnisse fragt, stellt man anhand der Antworten aus den Fragebogen des FOROST-Projekts (Rücklaufquote ca. $17 \%$ von ca. 2000 befragten Unternehmen) Folgendes fest: $45 \%$ dieser Unternehmen in Tschechien lassen im Rahmen der internen Kommunikation Dokumente übersetzen (bei den großen Unternehmen sind es sogar $65 \%$ ), wobei ca. ein Drittel davon explizit angegeben haben, dass es sich um Korrespondenz (einschl. Verträge) handelt. ${ }^{2}$ Hierzu kommen dann noch Übersetzungen in den Sprachrichtungen Englisch-Tschechisch, Deutsch-Englisch usw.

Hierbei ist es wichtig, sich vor Augen zu halten, dass die Firmen nicht nur professionelle Sprachvermittler fest anstellen oder (un)regelmäßig externe Übersetzer (bzw. Dolmetscher) benötigen, sondern dass immer häufiger auch anderweitig beschäftigte Mitarbeiter für diese Tätigkeiten eingesetzt werden. ${ }^{3}$ Im Hinblick auf den Untersuchungsgegenstand dieser Arbeit lässt sich (auch aufgrund von Aussagen von Mitarbeitern in den Unternehmen) wohl behaupten, dass man mittlerweile so gut wie keine Briefe oder E-Mails mehr von externen Sprachvermittlern übersetzen lässt. Dies geschieht, wenn überhaupt, betriebsintern.

Was die Sprachwahl bzw. den Einsatz eines Übersetzers (professionell oder nicht) betrifft, sind bei dem Erstellen von klassischen Briefen und von E-Mails einige Unterschiede zu vermuten, die u. a. aus dem Charakter des jeweiligen Schriftstückes resultieren. So ist davon auszugehen, dass das Erstellen eines papierenen Briefes mehr Aufwand darstellt. Es wird mehr auf die Muttersprache bzw.

1 Problemen dieser Art versuchen die Firmen bereits bei der Mitarbeitersuche/-einstellung vorzubeugen, indem sie Kandidaten mit Fremdsprachenkenntnissen bevorzugen oder gar fordern, vgl. die Analyse der Stellenanzeigen in NEKULA/MARX/ŠICHOVÁ (2009).

2 Da viele Dokumente, die nach außen gehen, auch noch Bestandteil der internen Kommunikation sind oder man die Art der Dokumente nicht immer eindeutig trennen kann, könnte auch (zumindest teilweise) die Anzahl derjenigen Unternehmen von gewisser Relevanz sein, die für externe Zwecke übersetzen lassen.

3 Fraglich ist dann, ob diese Unternehmen die Fragen im Fragebogen nach dem Übersetzen mit ja beantwortet haben - die Interviews oder das Nachfragen in informellen Gesprächen deuten darauf hin, dass dies eher nicht der Fall war. Dann wäre allerdings die Anzahl der Unternehmen, in welchen Übersetzungen eine Rolle spielen, wesentlich größer. Die Aufteilung der Sprachvermittler (Professionelle $\times$ Mitarbeiter mit einer anderen Funktion) in der Firma wie auch die genaue Frequenz des Übersetzens (für das Deutsche wie das Englische) gilt es noch zu untersuchen. 
Sprachkenntnisse des Adressaten geachtet, die sprachliche Richtigkeit des Textes und somit die der Übersetzung spielt eine größere Rolle, so dass bei unzureichenden Fremdsprachenkenntnissen ein Sprachvermittler eingesetzt wird.

Einiges deutet darauf hin, dass man, wenn man die Fremdsprache „nicht gut genug beherrscht", eher bereit ist, eine E-Mail in der Fremdsprache (ohne Sprachvermittler) zu verfassen als einen klassischen Brief. Die E-Mail kann als eine Art Kommunikationsstrategie eingesetzt werden: „[... [ written expression, often emails, can compensate for insufficient competence in the production and reception of spoken expression which is why it is sometimes used as a ,mild' form of avoidance strategy" (NEKVAPIL/SHERMAN: 2008, 10).

\section{Klassischer Brief und E-Mail: Verwendung, Sprache(n). Beispiele aus drei Unternehmen}

Nun soll die schriftliche innerbetriebliche Kommunikation durch die Untersuchung der Korrespondenz in drei konkreten Firmen in Tschechien illustriert werden.

\subsection{Unternehmen $X$}

Beim Unternehmen $\mathrm{X}^{1}$ können im Hinblick auf die Häufigkeit der Verwendung von klassischen Briefen und E-Mails wie auch hinsichtlich der Verwendung der Sprachen Tschechisch, Deutsch und Englisch in diesen Kommunikaten nur allgemeine Aussagen getroffen werden, da keine Briefe und E-Mails gesichtet werden konnten.

In der internen schriftlichen Kommunikation des Unternehmens X spielen laut Aussage eines deutschen Managers E-Mails die primäre Rolle.

Zitat 3 (informelles Interview Dt):

KŠ: Werden in der Firma in der innenbetrieblichen Kommunikation papierene Briefe verwendet?

Befr.: Nein. Fast alles läuft per Mail.

KŠ: Auch die ganz offiziellen Sachen?

Befr.: Ja, außer wichtige Verträge und so, Arbeitsvertrag, das muss unterschrieben werden.

$\overline{1 \text { Das Unternehmen } \mathrm{X}}$ ist eine große, ausschließlich an Produktion orientierte Firma mit einer großen deutschen Muttergesellschaft und befindet sich in einer kleinen tschechischen Stadt. Zum Zeitpunkt der Interviews waren dort ca. zehn ausländische Mitarbeiter (von insgesamt ca. 2000) beschäftigt. Näheres zur Kommunikation im Unternehmen X vgl. NEKULA/NEKVAPIL/ŠICHOVÁ (2005b) und NEKVAPIL/NEKULA (2006). 
Über die sprachliche Wirklichkeit im Unternehmen sagt Einiges auch die Tatsache aus, in welchen Sprachen die E-Mails verfasst werden. In dem Falle, dass eine Sprache nur von einer gewissen Gruppe von Mitarbeitern/Briefempfängern im Unternehmen verstanden wird, determiniert die Wahl der Sprache die Gruppe von potenziellen Adressaten bzw. Lesern des Briefes, vgl. NEKVAPIL/SHERMAN $(2008,10)$.

Die ausländischen Mitarbeiter erhalten selten E-Mails auf Tschechisch; falls ja, dann würde es sich „nie um etwas Wichtiges“ (Interview Dt) handeln - sie betrachten eine solche E-Mail nicht (oder nicht immer) als relevant genug, um sich damit genauso (schnell, ernst etc.) zu beschäftigen wie mit einer E-Mail auf Englisch oder Deutsch. Anders gesagt: In bestimmten Situationen ist die Sprache der E-Mail ein Indiz für die Wichtigkeit ihres Inhaltes. Die wichtigen Informationen werden an die deutschen/ausländischen Manager auf Deutsch oder Englisch kommuniziert, entweder direkt in der E-Mail vom Absender so verfasst, oder dann von einer Sprachassistentin übersetzt. ${ }^{1}$ Die deutschen/ausländischen Mitarbeiter selbst schreiben ihre E-Mails entweder auf Englisch, wenn der Text an mehrere (ggf. unbekannte) Personen bzw. mehrere Orte gelangen wird, oder (je nach Sprachkenntnis des/der konkreten Adressaten) auf Deutsch oder auf Englisch. Untereinander schreiben die Deutschen laut Aussage eines deutschen Managers ihre E-Mails in der Regel auf Deutsch. Die tschechischen Kollegen kommunizieren untereinander auf Tschechisch, falls der Text an einen ausländischen Kollegen geht, verwenden sie je nach Fremdsprachenkenntnissen Deutsch oder Englisch. ${ }^{2}$

Englisch wird in der schriftlichen Kommunikation (E-Mails) des Unternehmens X häufiger als in der mündlichen Kommunikation verwendet, ${ }^{3}$ unter anderem deswegen, weil man (laut Aussage eines deutschen Managers) mit der Fachterminologie auf

1 Laut Aussage eines Managers antwortet er auf tschechische E-Mails nicht immer. Wenn er einen wichtigen Inhalt vermutet oder nicht ausschließen kann, lässt er sich die E-Mail von seiner Assistentin informell übersetzen und fordert dann per E-Mail den Absender dazu auf, mit ihm fortan auf Deutsch oder Englisch zu kommunizieren. Hierzu vgl. auch NEKVAPIL/SHERMAN (2008, 10-11).

2 Offiziell wird seitens der deutsch-tschechischen Unternehmensführung versucht, auch im Falle der Brief- bzw. E-Mailkommunikation die Sprachsymmetrie zu unterstützen, indem es bei offiziellen und an alle Mitarbeiter gerichteten E-Mails, Briefen oder Aushängen entweder auf das Englische, welches für alle Kommunikationsteilnehmer eine Fremdsprache ist, zurückgegriffen wird, oder diese Texte zwei- oder dreisprachig auf Tschechisch, Deutsch, ggf. Englisch verfasst sind. Diese Strategie als eine der Möglichkeiten der Unternehmensleitung, die vorhandene sprachliche Asymmetrie im Unternehmen zumindest teilweise und nach außen hin auszugleichen, ist bei den deutsch-tschechischen Unternehmen neben der zweisprachigen Gestaltung der Homepage, der Informationen im Intranet und der Ausschilderung im Betrieb gerade bei den Briefen und E-Mails an die Mitarbeiter häufig zu beobachten.

3 Zum Beispiel in der schriftlichen Kommunikation des Unternehmens X mit der Mutterfirma in Deutschland kann man die größere Bedeutung des Englischen gegenüber dem Deutschen gut beobachten: Laut Aussagen der dortigen Mitarbeiter werden $30 \%$ der Briefe und Unterlagen der Tochtergesellschaft an die Muttergesellschaft auf Deutsch verfasst, in umgekehrter Richtung kommen nur $10 \%$ der Texte in deutscher Sprache. 
Englisch oft besser zurecht kommt als mit Deutsch, v. a. aber deswegen, damit die schriftlichen Unterlagen auch an weitere Betriebe weltweit (im Falle dieses Unternehmens X z. B. England, Frankreich, Brasilien) geschickt werden können, ohne sprachlich geändert werden zu müssen. Dies könnte v. a. für weltweit agierende multinationale Unternehmen gelten; im Unterschied dazu wird in mittelgroßen und kleinen deutsch-tschechischen Unternehmen in Tschechien innerbetrieblich die Rolle des Englischen kleiner sein. ${ }^{1}$

\subsection{Unternehmen $Y$}

An folgendem Beispiel sollen klassische Briefe und E-Mails in der Unternehmenskommunikation etwas ausführlicher im Hinblick auf ihre Formalien, aber auch die Handlungsdimension und Textfunktion skizziert werden. Für diesen Aufsatz wurden ca. 50 Briefe, E-Mails und Protokolle (Datierung: März bis Dezember 2000 und März bis Juni 2001) aus dem Unternehmen Y untersucht, einem in einer kleinen Stadt in Westböhmen angesiedelten Tochterunternehmen eines großen deutschen Konzerns. ${ }^{2}$ Nach der Analyse der Texte konnte eine Rücksprache mit dem Inhaber dieser Korrespondenz gehalten werden.

Der Adressat (direkt oder in Kopie) oder Absender der Briefe und E-Mails war einer der zwei deutschen Geschäftsführer.

Diese schriftlichen Nachrichten stellen sowohl Geschäftsbriefe dar, die zum Teil an externe Adressaten, vor allem aber an die Mutterfirma, oder intern verschickt oder empfangen wurden, als auch Briefe mit internen Mitteilungen oder Anweisungen der Geschäftsführung, der Personalabteilung usw. und die Korrespondenz mit dem Gewerkschaftsverband. Bei der Korrespondenz überwiegen erwartungsgemäß die Informationsfunktion und die Appellfunktion, manchmal ist auch die Obligationsfunktion zu finden, und zwar mit rein geschäftlichen Themen. Es ist davon auszugehen, dass halboffizielle E-Mails mit z. B. Kontaktfunktion oder Appellfunktion ebenfalls existieren/existiert haben, jedoch nicht archiviert worden sind.

Der deutsche Manager verfügte laut eigener Aussage zu diesem Zeitpunkt über sehr gute Französisch- und gute Englischkenntnisse, aber nur über rudimentäre

1 Sicherlich spielt hierbei aber auch die Branche und v. a. die Sprache der Kunden eine Rolle. So ergab zum Beispiel die Untersuchung in einer Prager Bank, der Tochtergesellschaft eines großen deutschen Bankunternehmens, dass das Deutsche die wichtigere Rolle spielt.

Zitat 4 (Interview Tsch):

Befr.: [...] und aber das meiste von der Korrespondenz oder von den internen Anordnungen oder irgendwelchen Vorschriften ist auf Deutsch. [...] Und teilweise auf Englisch.

2 Zu dem Zeitpunkt, als die Briefe und E-Mails entstanden, wurde das Unternehmen von ungefähr 2500 vorrangig tschechischen Mitarbeitern von zwei deutschen Geschäftsführern geleitet und war auf Produktion orientiert. Da die Anonymität und die Wahrung der firmeninternen Angelegenheiten meinerseits gewährleistet werden muss, können hier die Schriftstücke nicht abgebildet werden. 
Tschechischkenntnisse, so dass er nicht imstande war, Brieftexte auf Tschechisch $\mathrm{zu}$ erstellen oder $\mathrm{zu}$ verstehen. Seine tschechische Assistentin hatte gute Deutschkenntnisse und mittlere Englischkenntnisse und war u. a. für das Übersetzen oder Dolmetschen für ihre(n) Vorgesetzte(n) zuständig. Einige Briefe hatte auch die Leiterin der Personalabteilung, die sehr gut Deutsch sprach, übersetzt oder direkt auf Deutsch verfasst. Innerhalb dieses Briefmaterials befinden sich kaum Briefe oder E-Mails auf Englisch oder in einer anderen Sprache (1x ungarisch, 1x slowakisch), das meiste ist auf Tschechisch oder auf Deutsch verfasst.

Häufig wurden vom deutsch-tschechischen Unternehmen Y bei der externen, aber auch der internen Kommunikation (z. B. an den Mutterkonzern in Deutschland) Briefe per Fax verschickt. Hierzu gab es ein Vordruckformular mit dem Namen des Unternehmens, einer Überschrift (Fax-Mitteilung/Fax-Message) und jeweils auszufüllenden Feldern ${ }^{1}$ im Briefkopf auf Deutsch und Englisch (in dieser Reihenfolge), was darauf schließen lässt, dass es vom Mutterkonzern übernommen wurde. Der Body des Briefes wurde je nach Bedarf auf Tschechisch oder auf Deutsch geschrieben. Die Signatur (samt Registerangaben, Konto-Nummern etc.) ist stets auf Deutsch. Auffällig ist, dass, auch wenn diese Mitteilung an einen tschechischen Adressaten gesendet wurde (z. B. eine Einladung auf eine Pressekonferenz an tschechische Zeitungen) und der Text des Briefes vollständig tschechisch ist, dieser deutsch-englische Vordruck verwendet wurde.

Der Vordruck für die internen klassischen Briefe im Unternehmen ist auf Deutsch verfasst: Es sind die Felder An, Von; Bearbeiter; Tel. +Fax, Eingangsvermerke, Ihre Zeichen und Ihre Nachricht vom, Unsere Zeichen, Ort und Datum auszufüllen. Auch Briefe, die einsprachig auf Tschechisch oder zweisprachig auf Tschechisch und Slowakisch verfasst wurden, wurden auf diese Vordrucke geschrieben. In den Briefen, in welchen eine "interne Mitteilung“ an alle Mitarbeiter der Firma geschrieben wurde, sind zwei Sprachversionen in einem Brief zu finden: zuerst der Text auf Tschechisch, dann auf Deutsch. Da darunter auch Mitteilungen für die Produktion fallen und es nach Angaben des Respondenten unter den sog. Bluecollars kein deutschsprachiger Mitarbeiter beschäftigt war, lässt sich diese Zweisprachigkeit als eine Strategie interpretieren, die vorhandene kommunikative Asymmetrie durch bewusste Darstellung von Symmetrie zu kompensieren.

Die durchgesehenen Briefe bestätigen die Aussage des Managers, dass er seine Briefe entweder direkt auf Deutsch verfasst und abgeschickt hat oder aber dass er sie ins Tschechische übersetzen hat lassen und dann den tschechischen Text versendet hat.

1 Links oben untereinander: An/To, Name/Name, Abteilung/Department, Ort/Location, Telefon/Telephone, Fax/Fax, Seitenzahl incl. dieser Seite/Number of pages incl. this page, Von/From; rechts oben untereinander: Von/From, Name/Name, Abteilung/Departement, Ort/Location, Telefon/Telephone, Fax/Fax, Datum/Date. 
Bei der archivierten E-Mail-Korrespondenz ist Folgendes zu beobachten: Wenn es um eine sehr kurze Nachricht an einen tschechischen Adressaten geht, ${ }^{1}$ wird diese seitens des deutschen Geschäftsführers auf Tschechisch geschrieben, d. h. die (Sprach)assistentin übersetzt nach Diktat direkt in die Mail. Abgeschickt wurden diese E-Mails stets mit der Unterschrift des Managers, manchmal von seiner E-Mail-Adresse, manchmal jedoch von der der (Sprach)assistentin. Wenn die Länge des Brieftextes über eine solche kurze Mitteilung hinaus geht, wird eine E-Mail verschickt, in deren Body zuerst die auf Tschechisch geschriebene (wohl übersetzte) Nachricht steht, darunter dann (in aller Regel) der Text auf Deutsch. Dies lässt darauf schließen, dass der deutsche Text zur Übersetzung an die Sprachassistentin geschickt oder direkt auf ihrem Computer geschrieben wurde, sie ihn übersetzt und dann von ihrer Adresse abgeschickt hat. Hier zeichnen sich einige Vorteile des Mediums E-Mail ab: Jederzeit kann so die Korrektheit der Übersetzung überprüft werden, bzw. die E-Mail kann bei Bedarf auch noch an einen deutschsprachigen Mitarbeiter mit- oder nachgeschickt werden. Auch wird wohl das Archivieren der (wichtigen) Ausgangspost seitens des Emittenten eine Rolle gespielt haben.

Wie in der E-Mail-Kommunikation allgemein, scheint es auch bei der E-MailKommunikation des Unternehmens Y üblich zu sein, beispielsweise bei einer Antwort abschnittsweise die Textpassagen, auf die Bezug genommen wird (sog. direktes Quoting), oder den kompletten Text (sog. indirektes Quoting) aus der „alten“ E-Mail in die neue Nachricht zu übernehmen (Termini vgl. DÜRSCHEID: 2005, 89 f.), was die Verfolgung des Ablaufs der Angelegenheit erheblich erleichtert. Aus brieftheoretischer Sicht handelt es sich um ein interessantes, in der E-MailKommunikation häufig genutztes, jedoch nicht völlig unbekanntes Phänomen (z. B. direkte Zitate, Versuche der Rekonstruktion der Textbausteine des eigenen Briefes oder der Antwort darauf).

Beobachtet werden konnte bei der analysierten E-Mail-Korrespondenz noch, dass Subjekt/Betreff stets in derselben Sprache geschrieben wurde wie der Text.

Für die E-Mail-Kommunikation wird ein häufiges Eliminieren von Grußformeln und/oder Anrede festgestellt, vgl. DÜRSCHEID $(2005,92)$. Dies kann hier jedoch nicht pauschal bestätigt werden. Die meisten untersuchten E-Mails weisen übliche brieftechnische Gewohnheiten auf. So bleiben vor allem die Kontaktformeln am Anfang und am Ende des elektronischen Briefes erhalten (Gruß, Anrede, Abschiedformel, Unterschrift), auch weitere Konventionen (Dank für die Nachricht, Entschuldigung für eine verspätete Reaktion usw.) sind vorzufinden. Es können in der innerbetrieblichen Kommunikation jedoch einige E-Mails gefunden werden, in denen Anfangsgruß und/oder Anrede und/oder Abschiedsformel fehlen. Dies nimmt tendenziell dann zu, wenn die betreffende E-Mail in besonderem Maße dialogischen Charakter annimmt, also wenn die E-Mail im Hinblick auf den Kontext (und in der

1 Zitat 5 (Brief Tsch): Sehr geehrter Herr XX, danke für Ihren Brief vom XX in der Sache XX. In den nächsten Tagen werden wir den Sachverhalt überprüfen. Mit Grüßen XX. 
Regel auch zeitlich) direkt an die vorherige Nachricht anknüpft und von ihr auch Text(passagen) übernimmt (oft werden dann gleich mehrere E-Mails gewechselt).

Eine Sonderstellung innerhalb der E-Mails nimmt die Grußformel ein. Viele Nutzer von E-Mails richten mittlerweile für ihre Nachrichten eine automatische Erstellung der sog. Signatur ein, in der nicht nur Name (Unterschrift), Position und Kontaktdaten, sondern häufig auch bereits die Grußformeln vorformuliert sind. Dies ist auch bei Unternehmen der Fall (für bestimmte Zwecke sind sie dazu durch Richtlinien verpflichtet), wobei manchmal - um die Unternehmensidentität zu stärken - die Signaturen für alle Mitarbeiter, d. h. auch in mehreren Ländern, einheitlich gestaltet werden. Wenn die Mitarbeiter die eingestellte Version des Mailprogramms übernehmen, kann es passieren, dass die Sprache der Signatur (und eben oft auch die des Abschlussgrußes) nicht der Sprache des eigentlichen Briefes entspricht. Dies sollte m. E. aber nicht weiter interpretiert werden. ${ }^{1}$

Die Angaben der Befragten scheinen auch die bereits in der Forschungsliteratur bekannte These zu unterstützen, dass die Schnelligkeit und Unmittelbarkeit des Kontaktes per E-Mail auch linguistische Folgen hat, vgl. HLADKÁ $(2006,23)$ und weiter unten. Zur Illustrierung der Spontaneität von E-Mails (im Vergleich zum anderen Pol der Dichotomie - der Vorbereitung beim klassischen Brief) kann das folgende Beispiel aus dem Unternehmen Y (eine E-Mail mit Appellfunktion) gleich in mehreren Punkten dienen: Fehlen von Begrüßung, syntaktische Unkorrektheit am Ende des Satzes, Unterschrift nur mit dem Nachnamen (andere Korrespondenzstücke derselben Emittentin sind in aller Regel mit dem Titel Ing. und dem Vor- und Nachnamen unterzeichnet) und v. a. die Abkürzung des Abschlussgrußes, der sogar auf Deutsch gehalten ist ${ }^{2}$ zu MfG vgl. SIEVER (2006); zu dieser und ähnlichen Abkürzungen auch SCHLOBINSKI (2006).

Zitat 6 (E-Mail von der Personalleitung an eine Assistentin der Geschäftsführung, unter dem Text ist die zu übersetzende E-Mail samt Header übernommen, Tsch):

Kristino, prosim o preklad pro pana XX než prijedu kolem poledne.

$M f G$

XXová

1 Interessanter erscheint mir im Hinblick auf die Signatur das Bestreben mancher Mitarbeiter/ Unternehmen, die Signatur zwei-/mehrsprachig zu gestalten (und wenn ja, in welchen Sprachen; vgl. weiter unten Unternehmen Z).

2 Hierfür gibt es keine Erklärung, zumal sowohl die Adressatin als auch die Verfasserin tschechische Muttersprachlerinnen sind und diese E-Mail keine Signatur enthält. Womöglich war die Verfasserin unkonzentriert und/oder sehr in Eile und hat automatisch diese Abkürzung gewählt, weil sie im Zweifelsfalle auch davon ausgehen konnte, dass der Gruß verstanden wird. Im Tschechischen gibt es übrigens eine äquivalente Abkürzung für einen offiziellen Gruß nicht.

3 Kristina, ich bitte um Übersetzung für Herrn XX bevor ich komme gegen Mittag. MfG XXová [Nachname]. 


\subsection{Unternehmen Z}

Am Beispiel von Unternehmen $\mathrm{Z}$ können die gleichzeitige Verwendung von unterschiedlichen Sprachen in der schriftlichen Kommunikation und auch die Spezifika der E-Mail-Kommunikation im Vergleich zur Kommunikation mittels klassischen Briefen gezeigt werden.

Von diesem großen Unternehmen in Prag, der Tochtergesellschaft eines deutschen Konzerns, wurden Protokolle zu insgesamt 13 Fällen des E-MailWechsels gesichtet, sechs davon gehören zu der rein internen Kommunikation. ${ }^{1}$ Was die in diesen zwischen Prag und Deutschland (z. T. auch Venezuela, also von Tochtergesellschaften untereinander und Tochtergesellschaft-Muttergesellschaft) gewechselten E-Mails verwendeten Sprachen betrifft, können verschiedene Modi beobachtet werden. ${ }^{2}$

Häufig werden in den E-Mails die ursprünglichen Nachrichten in den neuen Brief mit übernommen, was das Verständnis der Sache auch für weitere Adressaten erleichtert. Bei jedem weiteren Verschicken können neue Adressaten hinzukommen, was auch genutzt wird. Ebenfalls wird häufig die interne Geschäftskorrespondenz an einige Personen in Kopie weitergeleitet. So ist man im Rahmen der E-MailKommunikation operativer und flexibler.

Wie bereits angedeutet, wird im Falle von E-Mails auf Grund der sog. Perzeption der Nähe bzw. durch größere Spontaneität mit dem Produzieren eines Textes anders umgegangen, was nicht nur für die Muttersprache gilt. Texte in der Fremdsprache werden wohl „leichter" verfasst und - wenn auch nicht ganz fehlerfrei - trotzdem verschickt, sobald man sich sicher ist, dass "der Sinn herauskommt“ (dies ist übrigens eine in vielen Unternehmen beobachtete Tatsache), wodurch man Zeit und Kosten spart. Gleiches kann man wohl auch für das Rezipieren voraussetzen, d. h. der Emittent schreibt eine E-Mail in seiner Muttersprache, wenn er weiß, dass der Adressat den Inhalt der Nachricht zumindest grob versteht, wenngleich er die

1 Ein Fall besteht jeweils aus mindestens zwei, maximal zehn E-Mails, wobei an die ursprünglichen Texte jeweils ein neuer anknüpft, die alten (aber nicht immer alle und nicht alle vollständig) bleiben Bestandteil der verschickten E-Mail. Herzlichen Dank an meinen Kollegen aus Prag, Herrn Oliver Engelhardt, der mir die aus seiner Untersuchung stammenden Protokolle betreffend der E-Mail-Korrespondenz der Firma $\mathrm{Z}$ zur Verfügung gestellt hat. Da ich jedoch die E-Mails selbst nicht durchsehen konnte, muss ich mich hier nur auf einige zusammenfassenden Aussagen und Interpretationen beschränken, die gegebenenfalls durch eine genaue Untersuchung revidiert werden würden.

2 Als Musterbeispiel für die sog. symmetrische Adaptation kann ein irgendwelche internen Absprachen enthaltender Mailwechsel zwischen zwei Mitarbeitern aus Prag betrachtet werden. Auf die von einem tschechischen Mitarbeiter auf Tschechisch verfasste und an einen deutschen Mitarbeiter adressierte E-Mail reagiert dieser auf Deutsch. In anderen Fällen wird die Sprache der E-Mail-Korrespondenz im Verlauf der Zeit im Hinblick auf den Empfänger (bzw. die Fremdsprachenkenntnisse des Absenders?) geändert. Ebenfalls finden wir den Fall der sog. Adaptation, in dem z. B von einem (tschechischen?) Muttersprachler eine auf Deutsch verfasste (fehlerhafte) E-Mail an den deutschen Leiter geschickt wird, oder die sog. Standardisierung, wo die Mitarbeiter in Venezuela den ganzen Brief (oder zumindest die Betreffzeile) auf Englisch schreiben. 
Sprache nicht perfekt beherrschen muss (auch hier verschiebt sich möglicherweise die Kommunikation in Richtung gesprochene Sprache). So können mehrere Sprachen in einem elektronischen Brief vorkommen, gar „vermischt" sein.

Als wichtig erscheint in der E-Mail-Kommunikation die Gestaltung der Betreffzeile, die auch als „Subjekt“ bezeichnet wird und zum Header der E-Mail gehört. Laut der sog. Netikette soll bei jeder E-Mail ein „Subjekt“ angegeben werden, was in der Geschäftskommunikation in der Regel auch der Fall ist. Vom Wesen her soll dieser Teil des elektronischen Briefes eine wichtige Kurzinformation über den Inhalt des Briefes geben ${ }^{1}$ und unterscheidet sich somit nicht von dem Betreff eines klassischen Geschäftsbriefs. Im Vergleich zu diesem ist jedoch der Text der Betreffzeile noch vor dem Öffnen des eigentlichen Brieftextes ersichtlich und kann somit sofort auf die Hauptfunktion (z. B. Kontaktfunktion bei „pozdrav “Gruß) oder auf das Hauptthema (z. B. „stižnost“/Beschwerde) hindeuten. In der interkulturellen Kommunikation spielt auch die Wahl der Sprache der Betreffzeile eine Rolle. Da das „Subjekt" einer schnellen Orientierung im Hinblick auf die Relevanz der E-Mail für den Adressaten dient, wird es häufig deswegen in der Sprache/den Sprachen verfasst, von der/den man ausgeht, dass der Leser bzw. die meisten (und zwar auch die potenziellen!) Leser sie verstehen, auch wenn sich diese Sprache von der Sprache des Bodys unterscheidet. So können wir z. B. im Unternehmen Z beobachten, dass eine spanisch geschriebene E-Mail mit einem Betreff auf Englisch versehen wird, wohl damit auch den Mitarbeitern in Europa, bei denen man keine Spanischkenntnisse automatisch voraussetzt/voraussetzen kann, sofort das Thema der Korrespondenz bekannt ist. Auch finden wir eine E-Mail auf Englisch mit einem deutschen Betreff. ${ }^{2}$ Meist scheint die Situation so zu sein, dass im Verlauf eines E-Mail-Wechsels die erste Betreff-Zeile übernommen bzw. im Bedarfsfall ergänzt wird. Auch so könnte erklärt werden, warum sich die Sprachen der Betreffzeile, des Bodys bzw. der Signatur voneinander unterscheiden.

Auch das Aussehen der sog. Signatur wäre im Hinblick auf die Sprachen von Interesse. Zum Beispiel gestalten manche Mitarbeiter des Unternehmens Z in Prag die Signatur zweisprachig, auf Tschechisch und Deutsch, manche Mitarbeiter des Unternehmens Z in Deutschland auf Deutsch und Englisch; beides in dieser Reihenfolge.

Aus sprachlicher Sicht ist noch der automatische Text des Mailprogramms relevant. Es ist jedoch davon auszugehen, dass dieser einmalig firmenweit eingestellt wurde und von den Mitarbeitern (außer in bewussten Ausnahmefällen)

1 Auch im Hinblick auf die permanent wachsende Anzahl von unerwünschten E-Mails (z. B. Spams) wird die Bedeutung dieses Feldes zunehmend größer, da sich manchmal bereits hier diese unerwünschten Nachrichten erkennen lassen.

2 Vom zweiten Adressaten wird die E-Mail auf Tschechisch geschrieben und die deutsche Betreffzeile übernommen, der dritte Schreiber schreibt seinen Text tschechisch, übernimmt die Betreffzeile und ergänzt sie auf Tschechisch. 
nicht von Fall zu Fall geändert wird. Daher lassen sich aus diesen Angaben nur bedingt Schlussfolgerungen zu Sprachpolitik oder Kommunikationsabläufen des Unternehmens ziehen. Allerdings könnte die (nicht)vorhandene Konvergenz solcher Formalien (Signatur, automatischer Text usw.) mit der deklarierten Firmensprache und anderen nach außen kommunizierten „Aussagen “ hinsichtlich Sprachsymmetrie interessante Impulse für die Unternehmensberatung bringen.

\section{Schluss: Klassischer vs. elektronischer Brief in den multinationalen Unternehmen}

Vor dem Hintergrund der aus den drei Unternehmen erworbenen Erkenntnisse wie auch nach der Analyse der vorhandenen Daten und der durchgeführten Gespräche können abschließend einige zusammenfassende Aussagen formuliert werden:

1. In der innerbetrieblichen Unternehmenskommunikation der deutschtschechischen Firmen wird sowohl der klassische als auch der elektronische Brief verwendet, wobei der papierene Brief weniger häufig bzw. zu anderen Zwecken (mit anderen Textfunktionen) verwendet zu werden scheint als die E-Mail. Laut Aussagen der Befragten werden alle wichtigen Briefe archiviert. Die meisten wichtigen geschäftlichen E-Mails werden ausgedruckt und archiviert, manchmal wird mit dem Ausdruck weiter gearbeitet.

2. Die interne Korrespondenz verläuft auf Tschechisch, Deutsch und/oder Englisch. In aller Regel werden Briefe von/an ausländische/n Mitarbeiter/n nicht tschechisch geschrieben. Bei unbekannten Adressaten richtet man sich nach dem Prinzip ,when you have to write something and you do not know whom it is for, do not use Czech“, so auch NEKVAPIL/SHERMAN $(2008,11)$.

3. Teile der (Geschäfts)korrespondenz werden übersetzt (E-Mails jedoch wesentlich weniger als papierene Briefe), und zwar in der Regel nicht von externen Sprachvermittlern. Unabhängig von der Art des Briefes und seiner Textfunktion werden auf jeden Fall (manchmal ausschließlich) Passagen oder Texte übersetzt, die Informationen von großer Wichtigkeit beinhalten. Somit kann die Zwei/Dreisprachigkeit der Dokumente auf ihre Relevanz hindeuten, und sei dies nur die deklarierte und vermittelte Kommunikations-symmetrie seitens der Unternehmensführung; vgl. NEKULA/NEKVAPIL/ŠICHOVÁ (2005b).

Zitat 7 (Interview Tsch):

Befr:: Also die Rundschreiben oder Texte, die grundsätzlich die ganze Filiale betreffen, die sind selbstverständlich zweisprachig. Weil wir trennen müssen zwischen dem Niveau der Sprachkenntnisse hier in unserer Abteilung 
oder in einigen Segmenten und dem der Sprachkenntnisse im Rahmen der ganzen Bank. Hier arbeiten selbstverständlich auch Mitarbeiter, die nur tschechisch sprechen. Ja. Es gibt gewisse Posten, für die man diese Fremdsprachenkenntnisse nicht braucht. [...]

$K \check{S}: M h m$.

Befr.: [...] zumindest die Bekanntmachungen, die hier zum Beispiel per Mail kommen, an alle Mitarbeiter, also das ist immer zweisprachig.

4. Auch E-Mails in der inneren Kommunikation der Unternehmen beinhalten wichtige geschäftliche Belange. Diese werden aber in der Regel wesentlich „spontaner“, d. h. auch mit weniger Zeitinvestition als entsprechende papierene Briefe verfasst. Bei den Muttersprachlern äußert sich das beispielsweise in einer geringeren Autokorrektur (und somit vermehrten Tippfehlern, Kleinschreibung usw. $)^{1}$ oder in angestrebter Schreibökonomie (vgl. SIEVER: 2006), im Hinblick auf die Fremdsprachen dann zum Beispiel darin, dass die tschechischen Mitarbeiter häufig direkt auf Deutsch oder auf Englisch und dann häufig nicht fehlerfrei schreiben und dies seitens der deutschen Leitung akzeptiert wird (aber sicherlich auch umgekehrt).

Zitat 8 (Interview Dt.):

Befr: Man akzeptiert in der Schriftsprache, dass manche Sachen halt, hm, also wenn, wenn Kollegen sich auf Deutsch äußern, etwas schreiben, zum Anfang hab ich gedacht, ich weiß, dass das sprachlich nicht richtig ist und so was, aber wenn ich es auf Tschechisch schreiben soll, wie würde das denn aussehen, also unterschreibe ich Dinge, die so eigentlich nicht korrekt sind, aber wo der Sinn klar rauskommt.

$K \check{S}: M h[\ldots]$

Befr: Das sind so die Dinge, die einfach bei der Übersetzung hinken, aber jeder weiß, was gemeint ist. Es verzehrt's auch nicht, es ist so ein bisschen der Spaß an der Sache. Genau, wenn ich Tschechisch spreche, dann kommen auch manche Dinge raus, das mit den sieben Fällen, und allen Endungen hinten dran, ja. [lacht].

Dies deutet darauf hin, dass sowohl die Absender als auch die Adressaten eventuelle sprachliche Unkorrektheiten als relativ unproblematisch betrachten und dass somit E-Mails als wenig „formal“ angesehen werden können.

1 Wobei die Textrezeption am Bildschirm flüchtiger als auf dem Papier ist, so dass bei Texten, die auf dem Computer verfasst/gelesen werden, grundsätzlich mit mehr Schreibfehlern zu rechnen ist, vgl. z. B. DÜRSCHEID $(2005,89)$. 
5. Außerdem scheint es, dass neben den rein offiziellen E-Mails mit professionellthematischer Ausrichtung diese Art Brief in der Unternehmenskommunikation auch zum Zweck der Gestaltung und Pflege von sozialen Beziehungen verwendet wird (näher vgl. die Ergebnisse des Projekts in schweizerischen Unternehmen von GÜNTHER KLEINBERGER/THIMM: 2000). Hier scheint aus funktionaler Hinsicht die E-Mail Situationen erobert zu haben, die vorher der mündlichen Kommunikation (Telefon, persönliches Gespräch) vorbehalten waren (z. B. Verabredungen mit Kollegen zum Essen oder zu Absprachen, Fragen, wie das Wochenende war usw.). Oft werden laut Aussagen der Befragten auch „persönliche Anliegen“ in eine geschäftliche E-Mail geschrieben, also Themen, die in einem papierenen Geschäftsbrief nicht vorkommen; es wird also noch eine zusätzliche Intension des Schreibers (Kontakt- und/oder Appellintention) deutlich und im hohen Maße zum Ausdruck gebracht und mehrere Themen vermischt.

6. Die Hauptintention des Briefschreibers und auch das Kontext, in dem E-Mails entstehen (Stichwort Quoting oder schneller Mailwechsel), haben zum Teil stark signifikante Auswirkungen auf den textuellen Merkmalbereich, das (Nicht)vorhandensein von Anrede- und Grußformel in den E-Mails usw., vgl. für klassische Briefe ERMERT $(1979,168)$.

7. Die alltägliche Nutzung der elektronischen Kommunikationssysteme (auch/oder insbesondere in der betrieblichen Kommunikation) wird dazu beigetragen haben, dass sich bei der E-Mail-Kommunikation einige sprachliche Unterschiede im Vergleich zum klassischen Brief herausgebildet haben. Wie bereits erwähnt, spielt die emotionale Dimension der Nähe, ein prototypisches Merkmal der gesprochenen Sprache, in E-Mails eine große Rolle. Obwohl dieses Medium durchaus geschriebene Sprache ist, beinhaltet es viele Formen der gesprochenen Sprache; Schriftlichkeit und Mündlichkeit beeinflussen sich stark. ${ }^{1}$ Das Auffälligste sind dabei die Verletzungen von Korrektheitsnormen der geschriebenen Sprache im weitesten Sinne, die toleriert werden, oder das ebenfalls tolerierte Nichteinhalten von Formalien (ausgelassene Anrede usw.). Manches kann jedoch nicht/nicht nur mit dem Merkmal Schriftlichkeit vs. Gesprochenheit erklärt werden. Gerade bei stark dialogischen E-Mails (mehrfacher Wechsel) kommt der Faktor der Spontaneität sehr gut zum Ausdruck. Auch Briefe, die an hochgestellte Mitarbeiter des Unternehmens per E-Mail verschickt werden,

1 Hierzu vgl. die Theorie der konzeptionellen und medialen Schriftlichkeit/Mündlichkeit von Koch und Oesterreicher, zitiert nach GÜNTHER KLEINBERGER/THIMM (2000, 6). Die konzeptionelle Ebene umfasst die „Modalität der Äußerungen unter dem Blickwinkel der damit verbundenen kommunikativen Strategie, die mit Gegensatzpaaren wie [...] formelle X informelle Sprache erfasst" wird. 
erhalten sprachliche Äußerungen, wie sie in einem klassischen geschäftlichen Brief nicht möglich wären.

Zitat 9 (E-Mail an die Personalleitung eines großen Unternehmens, Tsch): [...] hinsichtlich des $X X$ habe ich es Herrn $X X$ gesagt und ich soll ihnen ausrichten, dass... [...]

Diese Unterschiede scheinen aber nicht für die Unternehmenskommunikation spezifisch zu sein, vielmehr sind es gattungstypische Merkmale des elektronischen Briefes.

8. Häufig werden Teile oder ganze Texte von vorherigen E-Mails in das Body übernommen (sog. Quoting), ungeachtet der Sprachen, in welchen sie verfasst wurden. Auch wechselt man, stets der Pragmatik unterworfen, innerhalb einer E-Mail die sprachlichen Kodes (zwei oder mehrere Sprachen), was in einem klassischen papierenen Brief wohl unüblich ist. Besondere Aufmerksamkeit muss der oft mehrsprachigen Betreffzeile gewidmet werden.

Mit dem raschen Verbreiten der E-Mail und deren Etablierung in der alltäglichen privaten wie auch der wirtschaftlichen Kommunikation ist der klassische Brief also nicht verschwunden; dieses Medium wurde aber mit anderen Funktionen, Merkmalen und Konnotationen versehen. Auch in den multinationalen Unternehmen ersetzt die E-Mail keineswegs die herkömmlichen Briefe, auch nicht die Fax-Schreiben, Telefongespräche oder andere Formen der innenbetrieblichen Kommunikation. Es ist jedoch unumstritten, dass die E-Mail zu einer der wichtigsten Formen der Kommunikation geworden ist.

Genauere Erkenntnisse zu Inhalten, Sprachgestaltung oder Funktionen der E-Mails in der internen Unternehmenskommunikation gerade im Rahmen der multinationalen und somit mehrsprachigen Unternehmen, die dazu verhelfen könnten, diese Kommunikation z. B. durch geeignete Managementmaßnamen zu optimieren, sind in der Forschung allerdings immer noch ein Desiderat, welches es zu beseitigen gilt.

\section{Literatur:}

AMMON (1997): Ammon, Ulrich. Sprachkontakt in multinationalen Betrieben, in: Goebl, Hans/Nelde, Peter H./Starý, Zdenek/Wölck, Wolfgang (Hg.): Kontaktlinguistik: Ein internationales Handbuch zeitgenössischer Forschung. Berlin, New York: Walter de Gruyter, 1997, 852-857.

BUNGARTEN (1997): Bungarten, Theo. Mehrsprachigkeit in der Wirtschaft, in: Goebl, Hans/Nelde, Peter H./Starý, Zdenek/Wölck, Wolfgang (Hg.): Kontaktlinguistik: Ein internationales Handbuch zeitgenössischer Forschung. Berlin, New York: Walter de Gruyter, 1997, 414-421. 
DÜRSCHEID (2005): Dürscheid, Christa. E-Mail - verändert sich das Schreiben? In: Sievert, Torsten/Schlobinski, Peter/Runkehl, Jens (Hg.): Werbesprache.net. Sprache und Kommunikation im Internet. Berlin, New York: Walter de Gruyter, 2005, 85-97.

ERMERT (1979): Ermert, Karl. Briefsorten. Untersuchungen zu Theorie und Empirie der Textklassifikation. Tübingen: Max Niemeyer, 1979.

GLAHN (2005): Glahn, Richard. Kommunikation im globalen Geschäftsleben. Frankfurt/ Main: Peter Lang Verlag, 2005.

GREULE (2008): Greule, Albrecht. Thesenblatt zum Hauptseminar „Briefkommunikation“. Zusammenfassung (auf der Grundlage der Tischvorlagen). Ms., 2008.

GRÜN (2005): Grün, Karl. Der Geschäftsbrief. Gestaltung von Schriftstücken nach DIN 5008 , DIN 5009, DIN 676 u. a. Berlin: Beuth, 2005.

GÜNTHER KLEINBERGER/THIMM (2000): Günther Kleinberger, Ulla/Thimm, Caja. Soziale Beziehungen und innerbetriebliche Kommunikation: Formen und Funktionen elektronischer Schriftlichkeit in Unternehmen, in: Thimm, Caja (Hg.): Soziales im Netz. Sprache, Beziehungen und Kommunikationskulturen im Internet. Wiesbaden: Westdeutscher Verlag, 2000, 270-287.

HERINGER (2007): Heringer, Hans Jürgen. Interkulturelle Kommunikation. Tübingen: A. Francke Verlag, 2007.

HLADKÁ (2006): Hladká, Zdeňka. Současná soukromá korespondence. Korpusové zpracování a lingvistické využití. Unveröff. Habil., 2006.

MÖLLER/BRANDMEIER (2002): Möller, Joachim/Brandmeier, Michael (2002): Der Aufbau der Wirtschaftsbeziehungen zu Mittelosteuropa - Ergebnisse einer Befragung ostbayerischer Unternehmen, in: Möller, Joachim/Nekula, Marek (Hg.): Wirtschaft und Kommunikation. Beitrage zu den deutsch-tschechischen Wirtschaftsbeziehungen. Munchen: Iudicium, 2002, 29-50.

NEKULA/MARX/ŠICHOVÁ (2009): Nekula, Marek/Marx, Christoph/Šichová, Kateřina. Sprachsituation in Unternehmen mit ausländischer Beteiligung in der Tschechischen Republik, in: Sociolinguistica 23. Tübingen: Niemeyer, 2009, 53-85.

NEKULA/ŠICHOVÁ (2004): Nekula, Marek/Šichová, Kateřina. Sprache als Faktor der wirtschaftlichen Integration, in: brücken, 2004, Nr. 12, 317-335.

NEKULA/NEKVAPIL/SICHOVÁ (2005a): Nekula, Marek/Nekvapil, Jiří/Šichová, Kateřina. Sprachen in multinationalen Unternehmen auf dem Gebiet der Tschechischen Republik. München: Forschungsverbund Ost- und Südosteuropa (forost), 2005.

NEKULA/NEKVAPIL/ŠICHOVÁ (2005b): Nekula, Marek/Nekvapil, Jiří/Šichová, Kateřina. Sprachen in deutsch-tschechischen, österreichisch-tschechischen und schweizerisch-tschechischen Unternehmen: Ein Beitrag zur Wirtschaftskommunikation in der Tschechischen Republik, in: Sociolinguistica 19. Tübingen: Niemeyer, 2005, $128-143$.

NEKVAPIL/NEKULA (2006): Nekvapil, Jiří/Nekula, Marek. K jazykové situaci v nadnárodních podnicích působících v České republice, in: Slovo a slovesnost, 2006, Nr. 66, 83-95.

NEKVAPIL/SHERMAN (2008): Nekvapil, Jiří/Sherman, Tamah. Pre-interaction management in multinational companies in Central Europe, in: Current Issues in Language Planning, 10, 181-198.

NEUSTUPNÝ/NEKVAPIL (2006): Neustupný, Jiř̌́ V./Nekvapil, Jiří. Language Management in the Czech Republic, in: Baldauf, Richard B. Jr./Kaplan, Robert B. (Hg.): Language Planning and Policy in Europe, Bd. 2. Clevedon, Buffalo, Toronto: Multilingual Matters, 2006, 16-201.

NICKISCH (1991): Nickisch, Reinhard M. G. Brief. Stuttgart: Metzler, 1991. 
SCHLOBINSKI (2006): Schlobinski, Peter (Hg.). Von *hdl* bis *cul8r*. Sprache und Kommunikation in den Neuen Medien. Mannheim, Wiesbaden: DUDEN, 2006.

SIEVER (2006): Siever, Torsten. Sprachökonomie in den „Neuen Medien“, in: Schlobinski, Peter (Hg.): Von ${ }^{*} h d l^{*}$ bis ${ }^{*}$ culsr*. Sprache und Kommunikation in den Neuen Medien. Mannheim, Wiesbaden: DUDEN, 2006, 71-88.

STEIN (2002): Stein, Dieter. Sprache im Internet - Internet in Universität und Wirtschaft, in: Jahrbuch der Universität Düsseldorf [pdf], 2002.

ŠICHOVÁ (2008): Šichová, Kateřina. Zur Stellung der deutschen Sprache in der tschechischen Wirtschaft. Überlegungen zum Thema anhand der Situation in einer bestimmten Gruppe von Unternehmen in Tschechien, in: Acta Universitatis Carolinae - Studia Territorialia, 2008, Nr. 14, 219-238.

VANDERMEEREN (1998): Vandermeeren, Sonja. Fremdsprachen in europäischen Unternehmen. Untersuchungen zu Bestand und Bedarf im Geschäftsalltag mit Empfehlungen für Sprachenpolitik und Sprachunterricht. Waldsteinberg: Heidrun Popp Verlag, 1998.

VOLLSTEDT (2002): Vollstedt, Marina. Sprachenplanung in der internen Kommunikation internationaler Unternehmen. Studien zur Umstellung der Unternehmenssprache auf das Englische. Hildesheim: Olms, 2002. 